\title{
Impact de l'altération sur le bilan chimique des diatexites du Massif de l'Edough (Annaba, NE Algérien)
}

\section{Impact of weathering on the chemical balance of the diatexites from the Edough Massif (Annaba, NE Algeria)}

\author{
S. Hadj Zobir ${ }^{1}$
}

RESUME

La zone d'étude, constituée de diatexites, est située sur le flanc NE du massif de l'Edough (région de Annaba/ Algérie). Le massif, qui est l'un des plus arrosé d'Algérie (entre 750 et 1200 mm/an), se caractérise par un climat de type méditerranéen. Les diatexites montrent un faciès pétrographique et métamorphique homogène. Les minéraux primaires dominants sont le quartz, le feldspath potassique, l'oligoclase et la biotite alors que ceux d'altération sont représentés par des minéraux néoformés tels que la kaolinite, l'illite, la montmorillonite et dans une moindre mesure la séricite. A l'opposé de la minéralogie, la chimie des diatexites est hétérogène. Les analyses chimiques du matériel prélevé, du sommet vers la base des roches étudiées, révèlent une évolution progressive dans la composition chimique en terme de gains et pertes en $\mathrm{Ca}, \mathrm{Na}, \mathrm{K}, \mathrm{Mg}$, et en éléments traces. Les valeurs de l'index d'altération chimique CIA varient de 62,79 à 68,32. Celles de l'index de météorisation (CIW) varient de 63,92 à 70,79 et les valeurs de l'index de fraîcheur (IFRAIS) de 76,15 à 46,35. Ces résultats, plus des pertes au feu élevées (P.F > 2\%), ainsi qu'un rapport $\mathrm{Rb} / \mathrm{Sr}>1$ (en moyenne 1.74) montrent que la distribution des éléments chimiques est entièrement contrôlée par la météorisation et que les diatexites ont subi une altération de type bisiallitisation de longue durée, dominée par le lessivage de la roche primaire.

Mots clés: Altération, Météorisation, CIA, CIW, IFRAIS, Diatexites, Edough.

\section{ABSTRACT}

The studied section, located on the eastern part of the Edough massif (region of Annaba, Algeria), is characterized by a Mediterranean climate and the area is one of the most humid of Algeria (precipitation between 750 and $1200 \mathrm{~mm} /$ year). It consists of diatexites with homogeneous petrographic and metamorphic facies. The primary minerals are quartz, feldspar, oligoclase and biotite whereas weathering products are mostly represented by illite and montmorillonite. Although all samples comprise the same mineral composition, due to changes in the mode, the chemistry of this diatexites is very heterogeneous. The chemical analyses of the rocks reveal a progressive evolution in the composition in terms of gains and losses in $\mathrm{Ca}, \mathrm{Na}, \mathrm{K}, \mathrm{Fe}, \mathrm{Mg}$ and in trace elements. The values of the Chemical Index of Change $(\mathrm{CIA}=62.79-68.32$, of the Chemical index of Weathering $(\mathrm{CIW}=63.92-70.79)$, the Freshness Index (IFRAIS = $76-46.35$ ) and the high value of the loss of ignition (L.O.I > 2\%) as well as the Rb/Sr ratio $>1$ indicate that the distribution of the chemical elements along the section is entirely controlled by weathering (bisiallitisation alteration type) during a long time.

Keywords: Weathering, CIA, CIW, IFRAIS, diatexites, Edough.

\section{Introduction}

La nature des processus de transformations chimiques et minéralogiques, que se soit sous l'effet de changements de température et/ou de pression ou de la circulation de fluides (endogènes et/ou exogènes), s'exprime par l'apparition ou la disparition de phases minérales ainsi que par un transfert de

\footnotetext{
1 Université Badji Mokhtar-Annaba / Faculté des Sciences de la Terre / Laboratoire Sols et Développement Durable / BP 12, 23000 Annaba, Algérie. Email: shadjzobir@yahoo.fr
} 
matière. La distribution des éléments chimiques au cours de l'altération dépend de nombreux facteurs tels que la mobilité des éléments chimiques, la composition chimique et minéralogique, les propriétés physiques de la roche mère ainsi que des conditions climatiques (pluviométrie, température et autres). Les roches étudiées constituent un banc de diatexites à composition minéralogique très homogène. Les observations de terrain montrent que cette formation se caractérise par l'absence de profils d'altération proprement dits. Cependant on observe du toit vers le mur du banc un classement granulométrique diffus, une fracturation à intensité décroissante ainsi qu'une friabilité croissante de la roche. Toutes les observations suggèrent une altération sélective de la roche ainsi qu'un mouvement de matière à petite échelle. Dans le but de mieux cerner l'évolution de l'altération, nous nous proposons d'étudier le comportement de quelques éléments chimiques (majeurs, traces et terres rares) au cours de la météorisation des diatexites.

\section{Cadre géologique}

Le massif de l'Edough, constitué d'un ensemble de formations métamorphiques mis en contact tectoniquement, forme une structure antiforme de direction NE-SW (fig. 1a). On y distingue du bas vers le haut:le cœur de l'antiforme composé de diatexites (gneiss à biotite et gneiss oeillés à deux micas) datés Néoprotérozoïque (Hammor \& Lancelot 1998), avec parfois des bancs de leptynites et de marbres (Hilly 1962., Gleizes et al. 1988; AhmedSaid \& Leake 1993). Au-dessus des gneiss vient une unité composée de micaschistes à grenat, disthène, sillimanite et andalousite avec des bancs métriques de marbres. Une unité composée principalement de séricitoschistes, chloritoschistes et schistes graphiteux à intercalations centimétriques à métriques de quartzites coiffe le tout. Le complexe métamorphique ainsi que la couverture sédimentaire localisée principalement à l'Ouest du massif, ont été recoupés durant le Miocène par des magmas de composition acide à intermédiaire avec la mise en place de roches volcaniques. L'altération y est très développée et la configuration pétrographique ainsi que tectonique du massif a favorisé la circulation de fluides météoriques et/ou hydrothermaux qui ont très fortement modifié la composition des différentes lithologies. Cette altération a provoqué un profond changement de la composition minéralo- gique et chimique de la roche, qui s'est traduit par l'apparition de séricite et de minéraux argileux (illites, montmorillonite et kaolinite). Les nombreux indices métallifères (minéralisations pyrométasomatiques, principalement localisées dans les skarns de diffusion, les filons polymétalliques à sulfures du socle) attestent d'une activité hydrothermale en plus de celle météorique. Les roches étudiées, localisées sur le versant Ouest d'un bassin versant secondaire sont des diatexites (Hadj Zobir 2008).

L'affleurement est traversé par un important réseau fissural discordant au litage apparent. La fissuration, importante dans la partie sommitale, diminue avec la profondeur. Dans la partie supérieure de l'affleurement le grain est généralement plus grossier (jusqu'à $4 \mathrm{~mm}$ ) que dans la partie inférieure, ce qui confère à la roche un aspect finement lité. L'altération des minéraux ferromagnésiens confère à la roche une couleur allant du brun clair au brun foncé vers la base. Les échantillons montrent une composition minéralogique relativement homogène. Contrairement à la partie sommitale, la base de l'affleurement se caractérise par une faible fissuration ainsi que par une granulométrie fine. Les diatexites sont traversées dans la partie Nord par un pointement de 2 ha de roches ultrabasiquebasiques (péridotites et métabasites) (fig. 1b) fortement serpentinisées et altérées (Hadj Zobir et al. 2007).

\section{Cadre géomorphologique et climatique}

La zone d'étude, constituée de diatexites, est située sur le flanc N.E du massif de l'Edough (région de Annaba/ Algérie). Le massif a été décrit par Hilly (1962) comme «un ensemble de reliefs d'altitude modérée, véritable îlot montagneux ceinturé par la mer». Le massif se caractérise par de fortes pentes de ses versants. Le versant NW du massif est plus doux que celui NE, qui montre une très forte dénivellation. Globalement, l'Edough se caractérise par un climat de type méditerranéen humide. La température moyenne annuelle est de $16^{\circ} \mathrm{C}$ et les précipitations y sont abondantes dépassant souvent le mètre $(1200 \mathrm{~mm})$. Le massif comporte quatre bassins versants plus ou moins limités par les lignes de crêtes principales. Le réseau hydrographique y est très dense et le drainage de type dendritique (fig. 2a). Les fortes pentes et la nature de la roche ne permettent pas le ruissellement diffus et favorisent l'écoulement rapide, qui 

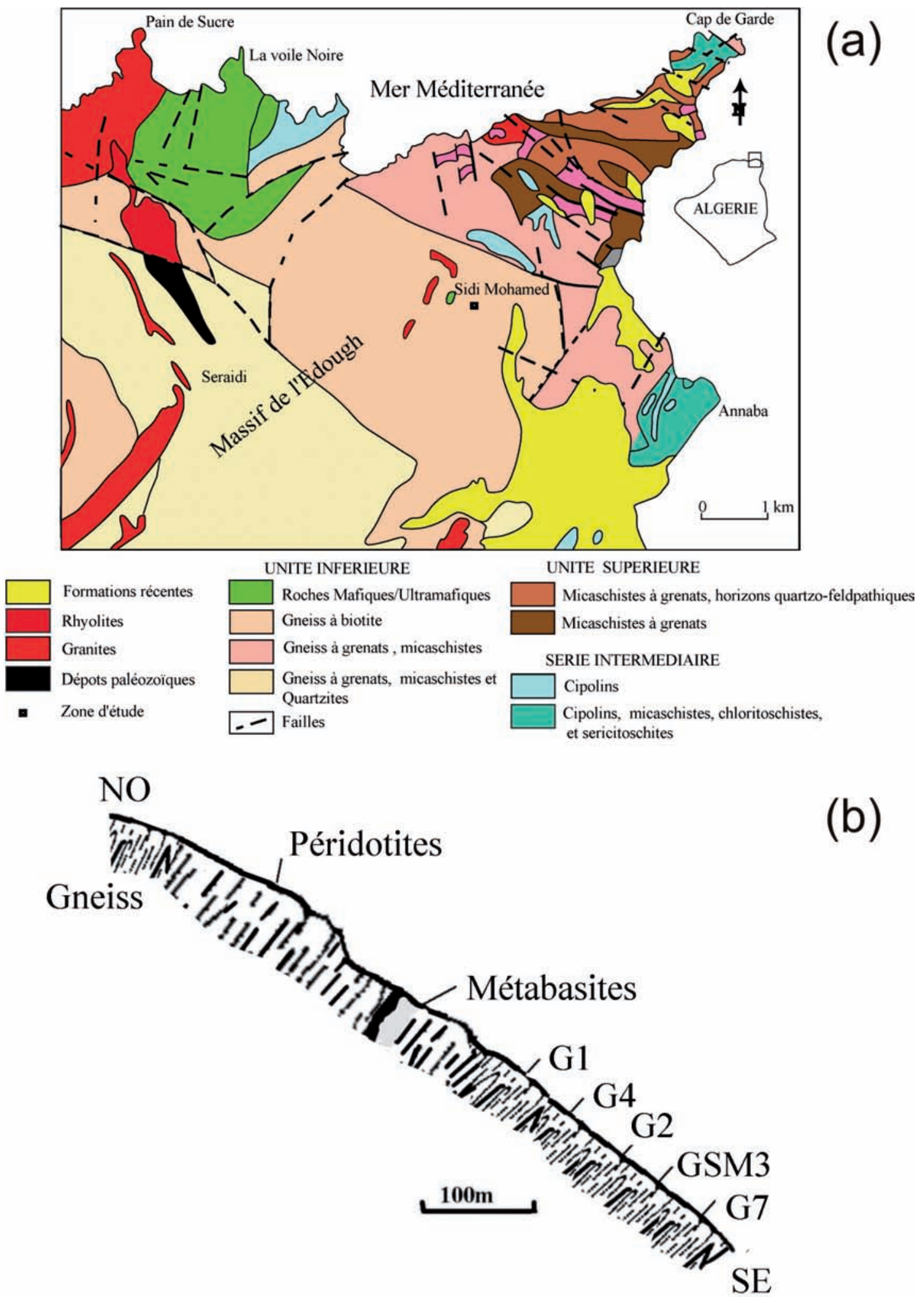

(b)

Fig. 1.-Cadre géologique du massif de l'Edough et de la zone étudiée ; (a) Carte géologique simplifiée du massif (modifiée sur la base des travaux de Hilly (1962), SONAREM (Société Nationale de la Recherche Minière) (1980), Gleizes et al (1988), Ahmed Saïd et al (1993)), (b) Coupe géologique NO-SE avec position des échantillons le long du profil de prélèvement, G1 : numéro de l'échantillon (l'échelle de la coupe au niveau des métabasites n'a pas été respectée dans le but de faire ressortir les détails). 


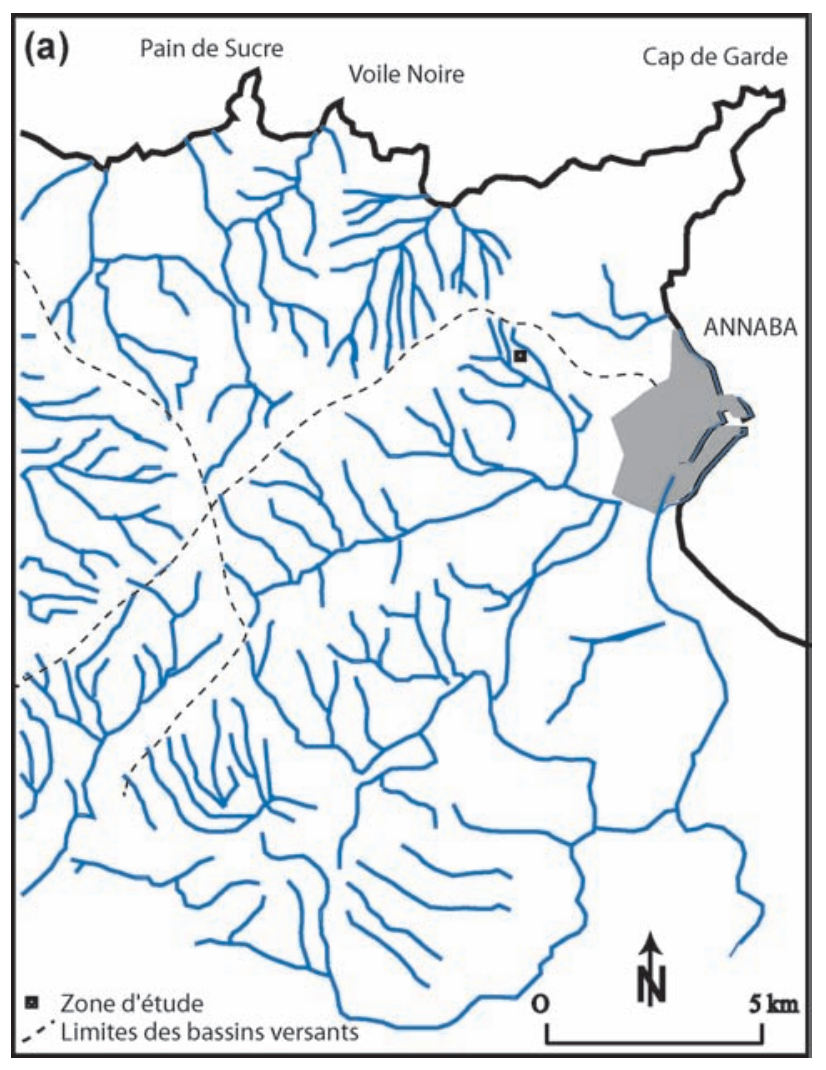

(b)

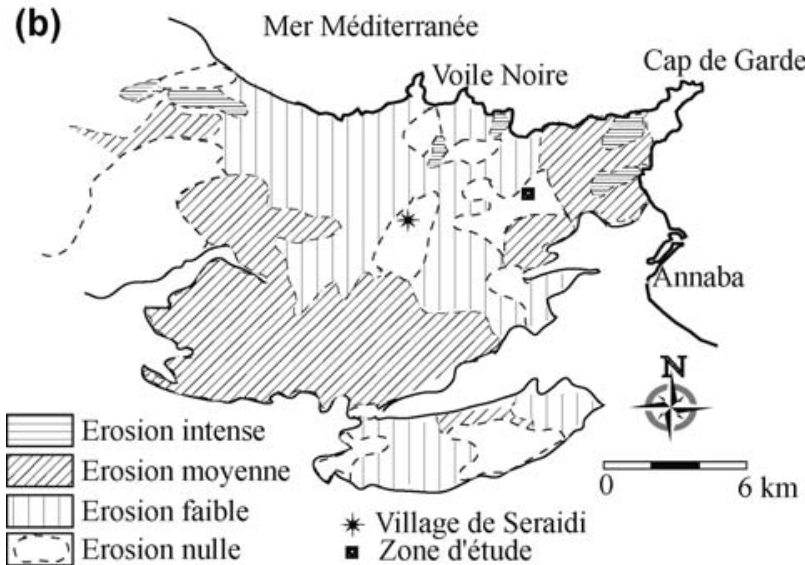

Fig. 2.-Cadre physique du massif de l'Edough; (a) Réseau hydrographique à drainage dendritique (Hilly, 1962); (b) Carte de sensibilité à l'érosion du massif de l'Edough (Oularbi \& Zeghiche, 2009)

peut atteindre $60 \%$ des précipitations (Oularbi \& Zeghiche, 2009), Ces caractéristiques topographiques et climatiques influent sur le degré de sensibilité à l'érosion du massif. Oularbi \& Zeghiche (2009) y distinguent quatre aires de sensibilités variables (fig. 2b). La zone étudiée, en pente avec des roches massives et cohérentes, comporte un couvert végétal important dans sa partie sommitale. Ces caractéristiques la place en zone à érosion nulle.

\section{Méthodes}

Les analyses des éléments chimiques ont été réalisées au laboratoire GeoForschungs Zentrum Potsdam (GFZ) et à l'Institut des Sciences de l'Université de Potsdam, Allemagne. Les éléments majeurs ont été analysés par un spectromètre à fluorescence $X$ automatique sur des pastilles préparées avec Spectromelt A12 (Merck). Les éléments traces et les terres rares ont été déterminés par ICP-MS (Plessen 1997; Grabe-Schönberg 1993) et ICP-AES (Zuleger \& Erzinger 1988). La détermination des minéraux argileux a été faite par radiocristallographie au laboratoire RX du Centre de Recherche et du Développement (CRD) de la SONATRACH, Boumerdès, Algérie.

\section{Description pétrographique et minéralogique}

L'aspect macroscopique des diatexites est folié avec parfois des faciès oeillés à grains grossiers feldspathiques. La roche est dans son ensemble cohérente, massive, de couleur claire à passées brunes. A certains endroits la roche montre une alternance de lits clairs et de lits sombres d'épaisseurs variables. Les niveaux clairs sont essentiellement quartzo-feldspathiques alors que ceux sombres sont riches en minéraux ferromagnésiens (biotite).

Au microscope, ces diatexites sont constituées de phénoblastes $(1$ à $4 \mathrm{~mm})$ de feldspaths (plagioclases et orthoclases), de quartz et de micas (biotite et muscovite) (fig. 3). L'orthose prédomine sur le plagioclase et les cristaux sont souvent craquelés et altérés (fig. 3a). Le plagioclase apparaît en phénoblastes de taille variable (2 à $5 \mathrm{~mm}$ ), maclé albite (fig. 3b). Les micas forment des plages, plus ou moins allongées, pouvant atteindre $1 \mathrm{~cm}$. Ils alternent avec des lits quartzitiques. La biotite est abondante, elle se présente en rubans de couleur brunâtre et est parfois ponctuée d'opaques (oxydes de fer). Les cristaux de biotites les plus altérés présentent un contour corrodé (fig. 3c) ainsi que des inclusions d'ilménite et de rutile. La muscovite se présente 

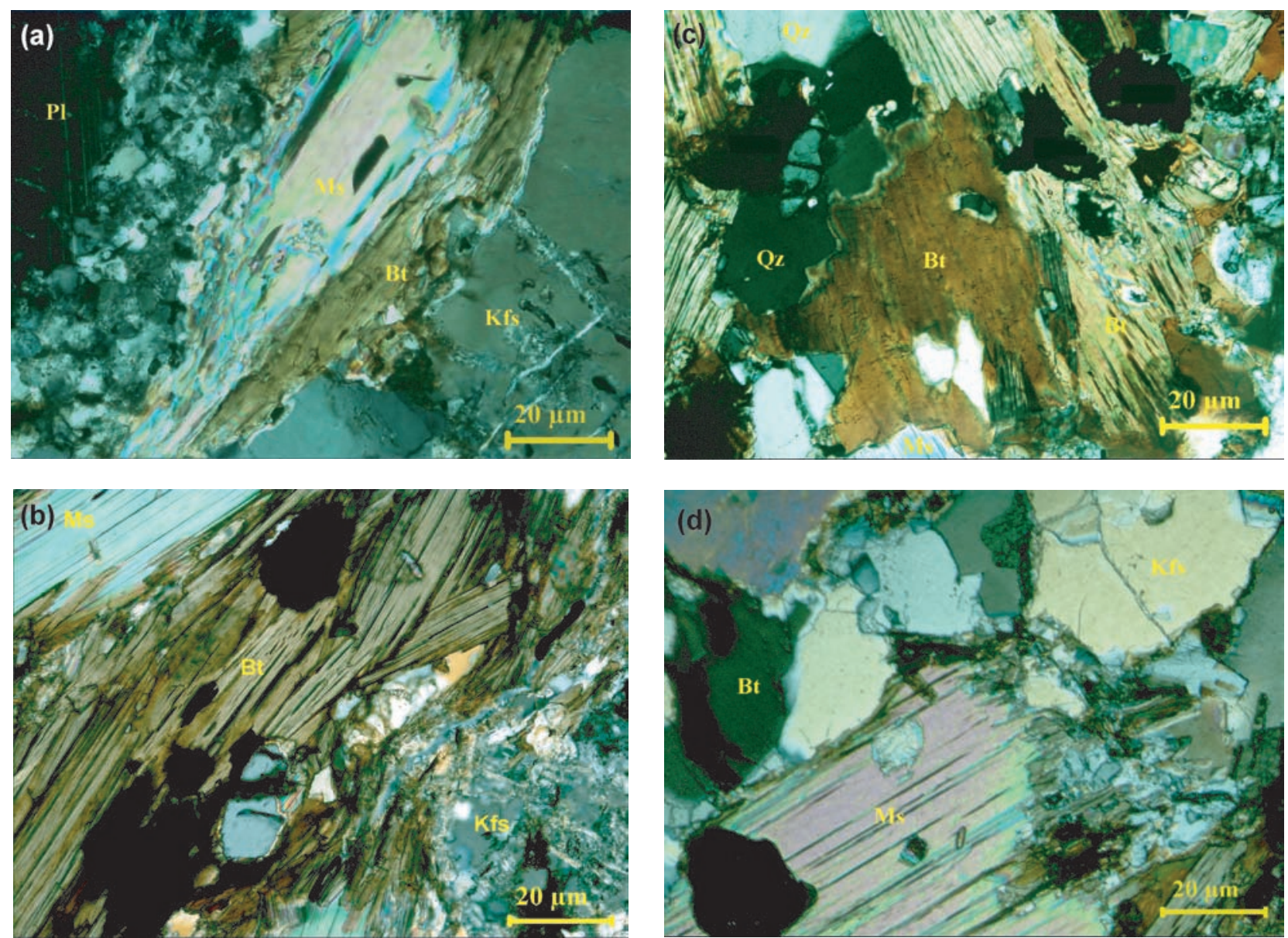

Fig. 3.-Photomicrographies en lumière polarisée analysée des diatexites; (a) Diatexites à porphyroblastes de feldspaths altérés; (b) Dans cette lame mince l'altération des feldspaths potassique est plus poussée, (c) Biotite fortement altérée et corrodée, (d) Muscovite altérée. Bt : biotite, Kfs : feldspath potassique, Ms : muscovite, Qz : quartz, PI : plagioclase.

également en lits sinueux et on la rencontre parfois en porphyroclastes à bordure fortement altérée et fragmentée (fig. 3d).

Les minéraux accessoires (apatite, zircon, ilménite et rutile) sont des minéraux ubiquistes dans les diatexites. L'apatite se présente en petits cristaux prismatiques alors que le zircon est sub-automorphe allongé et limpide. L'ilménite est présente, sous forme de chapelets dans les biotites, uniquement dans les échantillons les plus altérés de la partie basale de l'affleurement. Elle est localement associée au zircon et à l'apatite. Le rutile se présente sous forme de microcristaux associés aux biotites très altérées.

La présence de minéraux argileux a été confirmée par radiocristallographie (RX), il s'agit principalement d'illite, de montmorillonite et de la kaolinite, présente uniquement dans l'échantillon le moins altéré (G1) prélevé dans la partie sommitale de l'affleurement.

\section{Les indicateurs de l'altération chimique}

La configuration texturale et structurale de la zone étudiée permet de distinguer deux types de milieux favorisant la formation de minéraux argileux. D'une part, la partie sommitale qui se caractérise par une forte fissuration et une granulométrie grossière correspond à un milieu ouvert, à fort drainage, favorisant la formation de la kaolinite. D'autre part, la base du banc qui se caractérise par une faible fissuration et une granulométrie fine permettant la mise en place d'un milieu confiné à faible drainage favorable à la formation de minéraux tels que l'illite et la montmorillonite. Du point 
Tableau 1.-Concentration en minéraux argileux des diatexites en fonction de leur position dans la coupe de prélèvement, (K- Kaolinite, I- Illite, MMontmorillonite)

\begin{tabular}{|c|c|c|c|c|c|}
\hline \multirow{2}{*}{$\begin{array}{l}\text { Localisation } \\
\text { dans } \\
\text { I'affleurement }\end{array}$} & \multirow[b]{2}{*}{ Caractéristiques } & \multirow{2}{*}{$\begin{array}{c}(\%) \\
\text { Echantillons }\end{array}$} & \multicolumn{3}{|c|}{ Minéraux argileux } \\
\hline & & & K & I & M \\
\hline Sommet & $\begin{array}{l}\text { Diatexites } \\
\text { fraiches }\end{array}$ & G1 & Traces & 100 & Traces \\
\hline Centre & $\begin{array}{l}\text { Diatexites } \\
+/- \\
\text { altérées }\end{array}$ & $\begin{array}{c}\text { G4 } \\
\text { G2 } \\
\text { GSM3 }\end{array}$ & & $\begin{array}{l}90 \\
70\end{array}$ & $\begin{array}{l}70 \\
30\end{array}$ \\
\hline Bas & $\begin{array}{l}\text { Diatexites } \\
\text { très altérées }\end{array}$ & G7 & & 45 & 50 \\
\hline
\end{tabular}

de vue minéralogique, les effets de l'altération se traduisent par l'apparition de minéraux néoformés tels que la kaolinite, l'illite et la montmorillonite ainsi que par la présence d'oxydes et hydroxydes de fer. La distribution des minéraux argileux le long du profil de prélèvement, kaolinite au sommet, illite et montmorillonite vers la base, confirme la présence de deux milieux d'altération différents (tableau 1).

L'étude des lames minces montre que les principaux minéraux ayant subi l'altération sont les micas et les feldspaths. De point de vue minéralogique on remarque, de l'échantillon G1 (le moins altéré) à G7 (le plus altéré), une augmentation de la concentration en minéraux argileux ainsi que du degré d'altération des minéraux primaires. L'échantillon (G1) qui représente, dans cette étude, la roche originelle a montré des traces de kaolinite en plus de l'illite et de la montmorillonite. La présence de la kaolinite confirme le bon drainage de la roche mère. L'illite et la montmorillonite, qui sont très présentes dans la zone intermédiaire et basse des diatexites, traduisent respectivement une altération dans des conditions de mauvais et de très mauvais drainage (Millot, 1964; Tardy, 1969). La concentration en illite diminue vers la base de l'affleurement alors que celle en montmorillonite augmente. L'augmentation de la concentration en montmorillonite est corrélée aux variations texturales et structurales du banc des diatexites, à savoir la diminution de la granulométrie des roches et de l'intensité du réseau de fissuration vers la base des diatexites.

Les indicateurs chimiques de l'existence d'une altération se reflètent également par de fortes valeurs de la perte au feu (P.F > 2\%) (tableau 2), ainsi que par le comportement des éléments chimiques dans la roche. La distribution des éléments
Tableau 2.-Analyses des éléments majeurs et traces des diatexites du massif de l'Edough. CIA : Index d'altération chimique, CIW : Index de météorisation, IFRAIS : Index de fraîcheur, Densité : densité normative des roches

\begin{tabular}{|c|c|c|c|c|c|}
\hline & G1 & G4 & G2 & GSM3 & G7 \\
\hline $\mathrm{SiO} 2(\%)$ & 71,47 & 67,92 & 67,74 & 69,71 & 68,03 \\
\hline $\mathrm{Ti02}$ & 0,43 & 0,68 & 0,59 & 0,61 & 0,72 \\
\hline Al203 & 13,69 & 15,19 & 15,61 & 15,54 & 15,20 \\
\hline Fetot & 3,32 & 4,60 & 4,08 & 4,69 & 4,89 \\
\hline $\mathrm{MnO}$ & 0,01 & 0,05 & 0,04 & 0,08 & 0,05 \\
\hline $\mathrm{MgO}$ & 0,63 & 1,04 & 1,05 & 0,96 & 1,21 \\
\hline $\mathrm{CaO}$ & 1,52 & 1,52 & 1,44 & 1,62 & 1,60 \\
\hline $\mathrm{Na} 20$ & 3,02 & 2,30 & 2,04 & 2,52 & 2,05 \\
\hline K20 & 3,58 & 4,19 & 4,64 & 3,38 & 3,40 \\
\hline P205 & 0,23 & 0,23 & 0,19 & 0,23 & 0,23 \\
\hline P.F & 2,08 & 2,07 & 2,28 & 1,63 & 2,70 \\
\hline Total & 99,97 & 99,79 & 99,70 & 100,96 & 100,09 \\
\hline CIA & 62,79 & 65,48 & 65,78 & 67,39 & 68,32 \\
\hline CIW & 63,92 & 69,88 & 72,33 & 68,65 & 70,79 \\
\hline IFRAIS & 76,15 & 59,95 & 58,37 & 53,38 & 46,35 \\
\hline Densité normative & 2,70 & 2,73 & 2,73 & 2,74 & 2,75 \\
\hline $\mathrm{Cr}(\mathrm{ppm})$ & 297,00 & 123,58 & 143,59 & 261,54 & 96,53 \\
\hline Cs & 5,78 & 6,11 & 5,65 & 3,98 & 7,00 \\
\hline $\mathrm{Ba}$ & 485,00 & 447,13 & 639,42 & 454,11 & 454,43 \\
\hline $\mathrm{Rb}$ & 142,00 & 212,38 & 198,89 & 144,04 & 163,56 \\
\hline $\mathrm{Sr}$ & 195,00 & 102,42 & 108,66 & 114,94 & 90,10 \\
\hline $\mathrm{Ni}$ & 17,98 & 18,69 & 19,30 & 20,16 & 17,70 \\
\hline $\mathrm{La}(\mathrm{ppm})$ & 32,22 & 36,13 & 31,92 & 33,12 & 29,21 \\
\hline $\mathrm{Ce}$ & 72,74 & 74,21 & 68,20 & 70,21 & 58,71 \\
\hline $\mathrm{Nd}$ & 36,09 & 36,62 & 33,08 & 34,51 & 29,40 \\
\hline $\mathrm{Sm}$ & 8,55 & 7,78 & 7,15 & 7,45 & 6,80 \\
\hline $\mathrm{Eu}$ & 1,02 & 0,96 & 1,14 & 0,96 & 0,93 \\
\hline Gd & 8,87 & 7,62 & 7,07 & 7,40 & 7,31 \\
\hline $\mathrm{Tb}$ & 1,55 & 1,29 & 1,17 & 1,24 & 1,21 \\
\hline Ro & 1,41 & 1,54 & 1,33 & 1,38 & 1,53 \\
\hline $\mathrm{Er}$ & 3,28 & 4,03 & 3,52 & 3,55 & 3,95 \\
\hline Tm & 0,46 & 0,57 & 0,48 & 0,49 & 0,58 \\
\hline $\mathrm{Yb}$ & 2,53 & 3,77 & 3,17 & 3,21 & 3,59 \\
\hline $\mathrm{Lu}$ & 0,33 & 0,53 & 0,44 & 0,45 & 0,50 \\
\hline
\end{tabular}

chimiques le long du profil de prélèvement montre des profils en dents de scie (fig. 4a), suggérant une mobilité des éléments chimiques. La présence de l'altération, quel que soit sa nature, se manifeste également par une distribution en éventail le long de droites d'altération dans le diagramme binaire $\mathrm{Al}$ versus Ti. Plus l'éventail est ouvert, plus l'altération est importante. La figure (fig. 4b) montre que les diatexites ont subi une altération modérée.

\section{Discussion}

Dans le but de déterminer la part de l'altération météorique de celle hydrothermale, trois index différents d'altération ont été calculés. Le taux d'alté- 
(a)
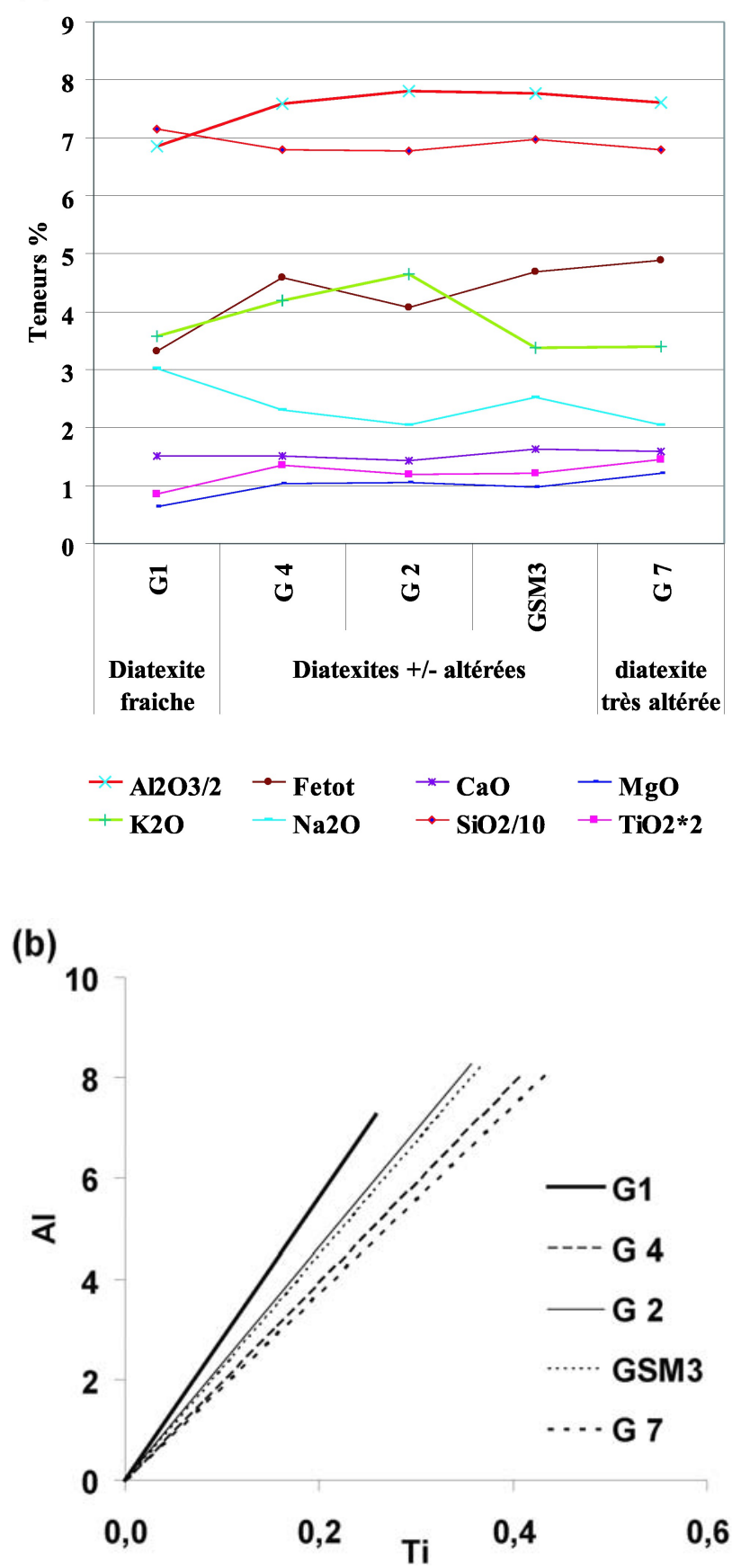

Fig. 4.-Diagrammes binaires montrant la mobilité des éléments chimiques; (a) Distribution des éléments chimiques en dents de scie suggérant leur mobilité dans l'affleurement, (b) Distribution en éventail le long de droites d'altération dans le diagramme binaire $\mathrm{Al}$ versus $\mathrm{Ti}$ indiquant une altération modérée. ration hydrothermale a été déterminé à partir de l'index de fraîcheur (IFRAIS) (Piché 2000, 2001). Si FRAIS $=0$ la roche est totalement altérée et si IFRAIS = 100 la roche est totalement fraîche. L'index est déterminé par la méthode NORMAT, développée par Piché $(2000,2001)$. Cette méthode est établie autour d'un logiciel de calcul des minéraux normatifs. Les valeurs d'IFRAIS varient de 76,15 (échantillon G1) à 46,35 (échantillon G7). Dans nos échantillons l'altération hydrothermale s'exprime principalement par la présence de séricite normative. L'altération météorique qui est un phénomène omniprésent dans le massif de l'Edough se reflète par la décomposition des minéraux silicatés, en formant des minéraux argileux tels que l'illite et la montmorillonite en plus de la séricite (déterminée par RX). L'index d'altération météorique CIW (Harnois 1988) met en relation l'aluminium, le calcium et le sodium par l'équation $\mathrm{CIW}=$ $\left[\mathrm{Al}_{2} \mathrm{O}_{3} /\left(\mathrm{A}_{2} \mathrm{O}_{3}+\mathrm{CaO}+\mathrm{Na}_{2} \mathrm{O}\right)\right] \times 100$ (où $\mathrm{Al}_{2} \mathrm{O}_{3}$, $\mathrm{CaO}$ et $\mathrm{Na}_{2} \mathrm{O}$ sont exprimés en proportions molaires). Plus l'index se rapproche de 100, plus le degré d'altération de la roche est élevé. Dans les diatexites, CIW varie de 63,92 (échantillon G1) à 70,79 (échantillon G7). Les valeurs des index d'altération, IFRAIS et CIW, montrent que les diatexites ont subi une altération modérée. Hormis la séricite normative et celle déterminée par RX, on note l'absence de minéraux hydrothermaux et la richesse des roches en montmorillonite. La prédominance modale des minéraux argileux par rapport à la séricite suggère que les diatexites ont subi, principalement, une altération météorique. L'index d'altération chimique CIA, $\left(\mathrm{CIA}=\mathrm{Al}_{2} \mathrm{O}_{3} /\left(\mathrm{Al}_{2} \mathrm{O}_{3}\right.\right.$ $+\mathrm{CaO}+\mathrm{Na}_{2} \mathrm{O}+\mathrm{K}_{2} \mathrm{O}$ ) (Nesbitt \& Young 1984), est également un bon indicateur du degré d'altération dans le cas de roches à composition chimique homogène.. Cet index qui a été calculé pour tous les échantillons varie de 62,79 (diatexites fraiches) à $68,32 \%$ (diatexites altérées) (tableau 2). Ces valeurs $>50$ indiquent que les diatexites sont constituées de matériel quartzo-feldspathique et qu'elles sont riches en minéraux d'altération (Nesbitt 1979; Nesbitt \& Young 1984). Cette variation traduit également l'évolution progressive de l'altération, ainsi que l'introduction progressive de minéraux argileux du sommet vers la base de l'affleurement.

La présence de la montmorillonite, ainsi que les valeurs (7.5 en moyenne) du rapport moléculaire $\mathrm{Ki}$ $\left(\mathrm{Ki}=\mathrm{SiO}_{2} / \mathrm{Al}_{2} \mathrm{O}_{3}\right)$ supérieures à 3, (Pédro 1966; Tardy 1969) indiquent la prédominance de la bisiallitisation comme processus l'altération. 

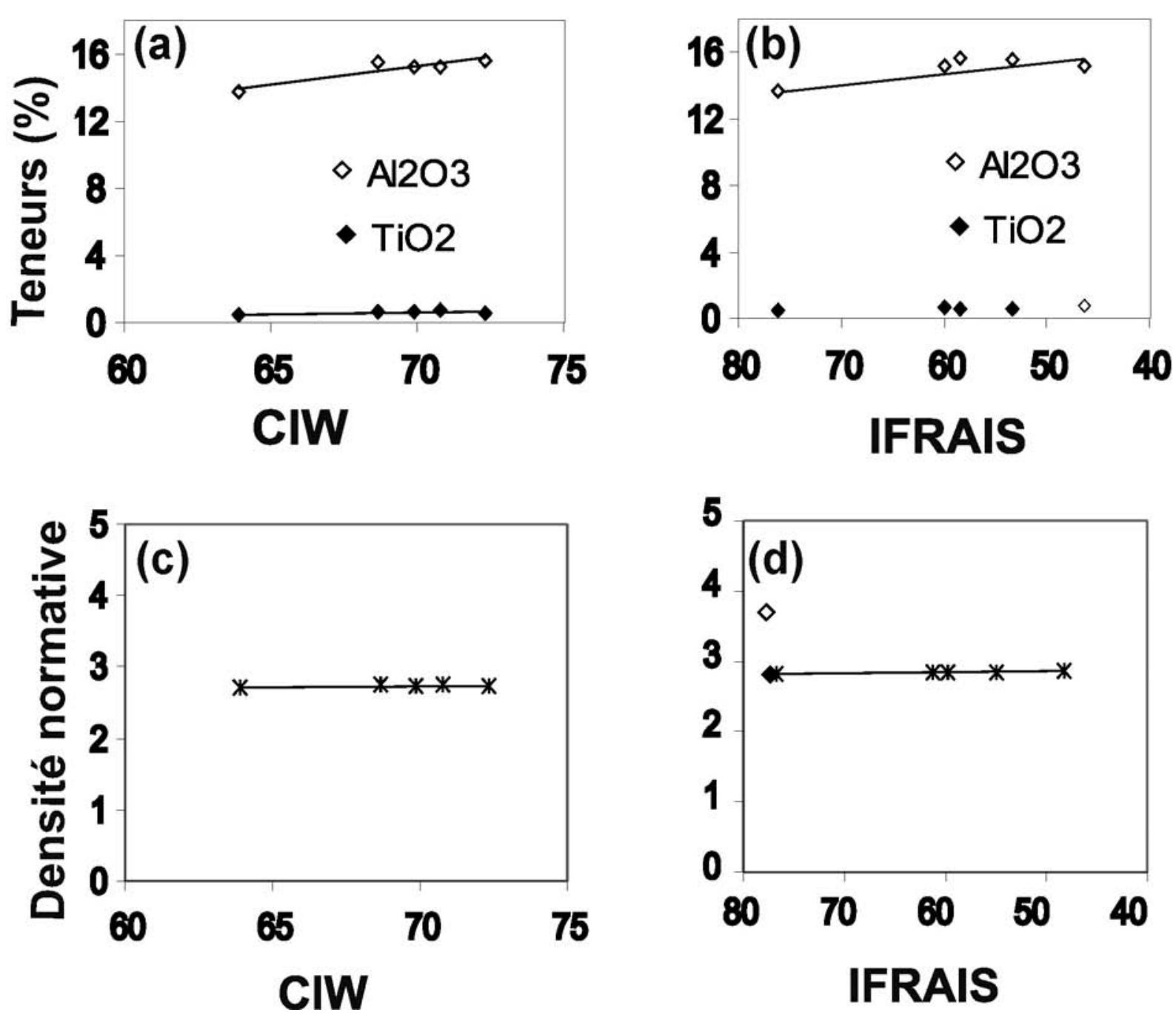

Fig. 5.-Distribution et évolution des éléments chimiques et de la densité normative des diatexites en fonction des index d'altération Clw, IFRAIS; (a) et (b): la tendance est que l'aluminium augmente avec le taux d'altération alors que la stabilité du titane n'est pas affectée par le taux d'altération, (c) et (d): le transfert des éléments chimiques s'est fait sans variation de la densité normative indiquant une altération isovolumique.

\section{Evolution chimique}

Durant l'altération, les études ont montré que le lessivage et la mobilité des éléments tels que le calcium, le sodium et le potassium sont proportionnels aux taux d'altération (Wronkiewicz \& Condie 1987). Ces éléments sont préférentiellement lessivés (Nesbitt \& Young, 1982). L'aluminium et le titane sont souvent considérés comme inertes dans les systèmes d'altérations (Gresens 1967; Grant 1986; Potdevin \& Caron 1986; Potdevin \& Marquer 1987). Cependant Gardner (1980) et Baldeyrou et al. (2003) ont démontré que l'aluminium peut être mobilisé dans certains types d'altérations, particu- lièrement dans les systèmes hydrothermaux. L'aluminium est mobile s'il est associé aux alcalins. Il permet la formation de phyllosilicates alumineux (Baldeyrou et al. 2003). La mobilité de l'aluminium et du titane au cours de l'altération des diatexites étudiées a été testée sur des diagrammes binaires: éléments versus l'index d'altération météorique (CIW) et hydrothermal (IFRAIS). Les figures 5a et $5 \mathrm{~b}$ montrent que l'aluminium est mobile et que sa concentration augmente avec le taux d'altération alors que le titane ne semble pas être affecté. Dans notre étude le titane est donc considéré comme élément immobile et a été choisi comme élément de référence dans la quantification des pertes et gains 
(a)
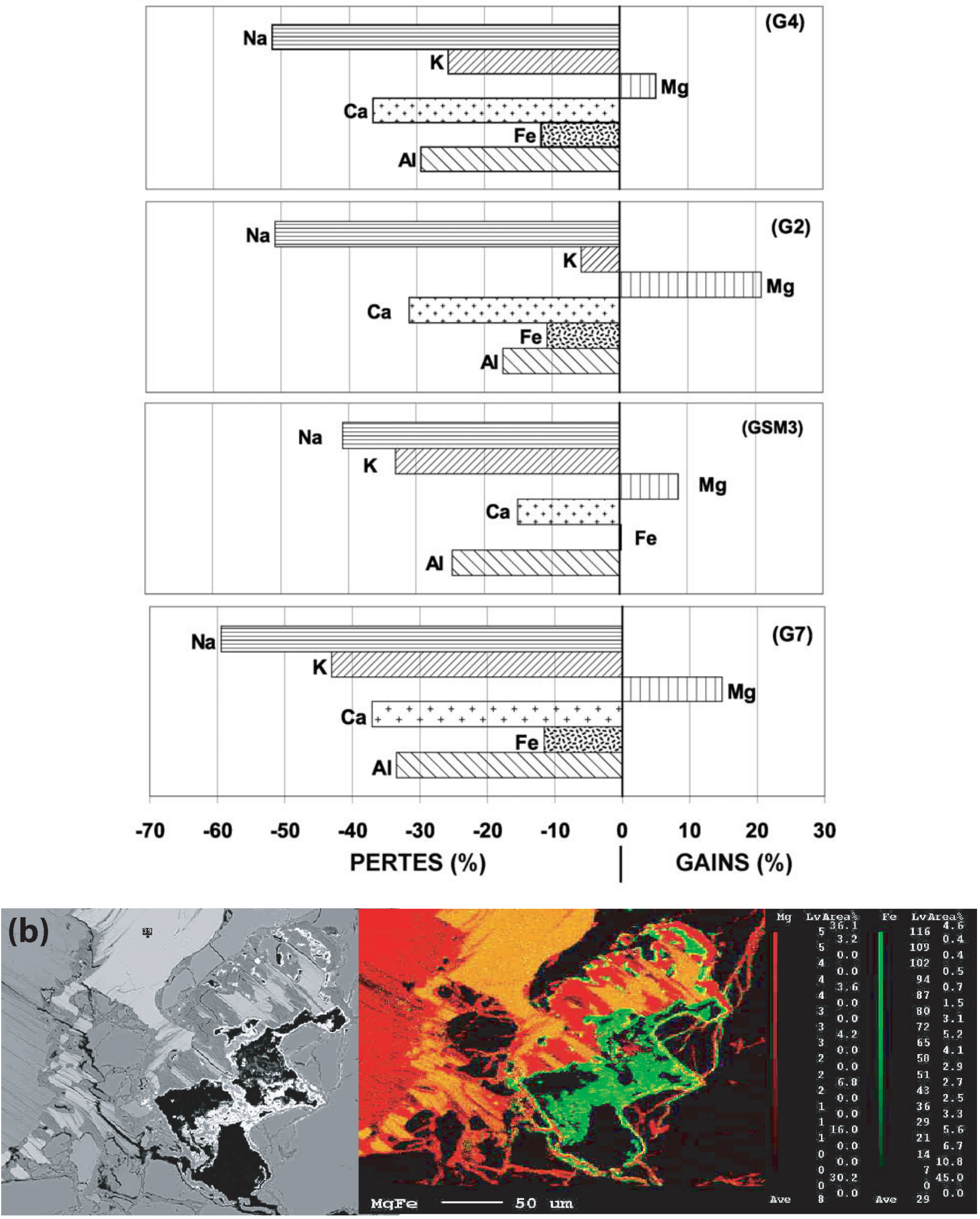

Fig. 6.-Distribution des éléments chimiques majeurs au cours de l'altération météorique des diatexites; (a) Diagrammes des gains et pertes en éléments majeurs, (b) Cartographie des éléments chimiques $\mathrm{Fe}$ et $\mathrm{Mg}$ dans une biotite altérée. 
en éléments chimiques au cours de l'altération. Le transfert des éléments chimiques (Al et Ti) s'est fait sans variation de la densité normative (Piché 2000, 2001) des roches (fig. 5c et 5d), indiquant une altération isovolumique.

Le tableau 2 liste les résultats des analyses chimiques en majeurs et traces. De la roche la moins altérée $(\mathrm{G} 1)$ à la plus altérée $(\mathrm{G} 7)$, les teneurs en $\mathrm{Al}_{2} \mathrm{O}_{3}$ évoluent de 13,69 à $15,2 \%, \mathrm{TiO}_{2}$ varie de 0,43 à $0,72 \%, \mathrm{MgO}$ de 0,63 à $1,21 \%, \mathrm{Fe}_{\text {total }}$ de 3,32 à $4,89 \%$ et $\mathrm{CaO}$ varie de 1,5 à $1,6 \%$. Les concentrations en $\mathrm{Na}_{2} \mathrm{O}$ évoluent de 3,02 à $2,05 \%$, alors que celles en $\mathrm{K}_{2} \mathrm{O}$ varient de 3,58 à $3,4 \%$.

L'évaluation du comportement et de la mobilité des éléments chimiques durant l'altération, en terme de gains et de pertes en un élément par rapport à la roche mère, s'est faite sur la base du pourcentage de modification $\left(\%\right.$ modification $=\left[\left(\mathrm{X} / \mathrm{TiO}_{2}\right)_{\text {Echantillon }^{-}}\right.$ $\left.\left.\left(\mathrm{X} / \mathrm{TiO}_{2}\right)_{\text {mère }}-1\right] \times 100\right)$ avec $\mathrm{X}=$ élément chimique mobilisé (Middelburg et al. 1988). La roche mère correspond à la roche la moins altérée (échantillon G1) alors que l'échantillon le plus altéré (G7) correspond à la roche finale après altération.

La figure $6 \mathrm{a}$ montre que $\mathrm{Al}_{2} \mathrm{O}_{3}$ enregistre une perte progressive variant de 17 jusqu'à $33 \%$, alors que celle de $\mathrm{CaO}$ est de $32 \%$ en moyenne. La valeur la plus importante, en termes de perte est celle de $\mathrm{Na}_{2} \mathrm{O}(60 \%)$. Les éléments calcium et sodium sont également lessivés de la roche parentale (G1). Le potassium montre une perte variant de 25 à plus de $40 \%$. Cette variation est en accord avec l'augmentation de la valeur de l'index d'altération. On note pour le magnésium un gain variant de 5 à $20 \%$ et les pertes en fer sont de l'ordre de $10 \%$. L'aluminium est progressivement lessivé de la roche parentale avec une augmentation de sa concentration vers la base du banc des diatexites. Cette augmentation traduit un accroissement du taux de la météorisation ainsi qu'une concentration plus importante de minéraux argileux alumineux dans l'affleurement. La mobilité du titane n'est pas affectée par l'altération. Les variations des teneurs en cet élément ne peuvent être attribuées à l'effet du taux d'altération et semblent être contrôlées par les phases titanifères (ilménite, rutile) et ferromagnésiennes plus abondantes à la base du banc et/ou sont le résultat d'un remaniement mécanique contrôlé par la topographie. Le magnésium et le fer ont un comportement différent de celui des autres éléments majeurs. Les fortes concentrations en $\mathrm{MgO}$ et en $\mathrm{Fe}_{2} \mathrm{O}_{3}$ dans les roches les plus altérées (échantillon $\mathrm{G} 7$ ) coïncident avec la prépondérance de la biotite primaire, des minéraux argileux et des oxydes/hydroxydes secondaires. Le magnésium n'est que très faiblement affecté par le processus de lessivage. L'augmentation de sa teneur, dans la roche très altérée, est à attribuer à une altération in situ, sous faible drainage, de la biotite primaire par lessivage du fer qui s'accumule pour former des minéraux secondaires tels que les oxydes et hydroxydes (fig. 6b). La présence de ces derniers se traduit, sur le terrain, par la couleur brunâtre et tachetée des roches fortement altérées.

Le taux de lessivage du calcium est homogène, il est préférentiellement incorporé dans les phyllosilicates pour former des smectites calciques tel que la montmorillonite. Le potassium et le sodium proviennent du lessivage des feldspaths. Le sodium montre un plus fort taux de lessivage que le potassium ce qui implique une importante séricitisation des plagioclases.

Le comportement des éléments traces peut être influencé par le métamorphisme, l'érosion et par l'altération qui peut être d'origine météorique ou hydrothermale. De part leur faible solubilité, les terres rares ne sont pas affectées par ces phénomènes. L'homogénéité pétrographique, minéralogique et thermobarométrique de l'affleurement de diatexites suggère un même degré de métamorphisme. La présence de phases argileuses en concentration croissante du sommet vers le bas de l'affleurement et les valeurs élevées de la perte au feu (P.F $>2 \%$ ) nous permettent de retenir, comme facteur principal influant la variabilité chimique de ces roches, l'altération par l'eau (météorique, de ruissellement et d'infiltration). Le $\mathrm{Cr}$ et le $\mathrm{Ni}$ montrent une faible variation des teneurs (tableau 2). Les valeurs élevées en ces éléments sont à attribuer à la présence proximale de roches ultrabasiquesbasiques (Hadj Zobir et al. 2007). Les profils de variation des éléments traces tels que $\mathrm{Ba}, \mathrm{Rb}, \mathrm{Cs}$ et $\mathrm{Sr}$ montrent des variations d'amplitude moyenne (fig. 7a). Le $\mathrm{Cs}, \mathrm{Rb}$ et $\mathrm{Ba}$ sont préférentiellement fixés par les minéraux argileux et sont moins lessivés que le $\mathrm{Sr}$. Ce dernier montre une chute très importante des teneurs, ce qui traduit sa forte mobilisation lors des différents processus d'altération ayant affecté les diatexites. Le rapport $\mathrm{Rb} / \mathrm{Sr}$ qui est supérieur à 1 (en moyenne 1,74), sauf pour l'échantillon le moins altéré $\mathrm{G} 1(\mathrm{Rb} / \mathrm{Sr}=0,73)$, indique un fort taux d'altération durant un long laps de temps (McLennan et al. 1983b).

Les teneurs moyennes globales en terres rares sont de l'ordre de $150 \mathrm{ppm}$, ce qui correspond aux 
(a)
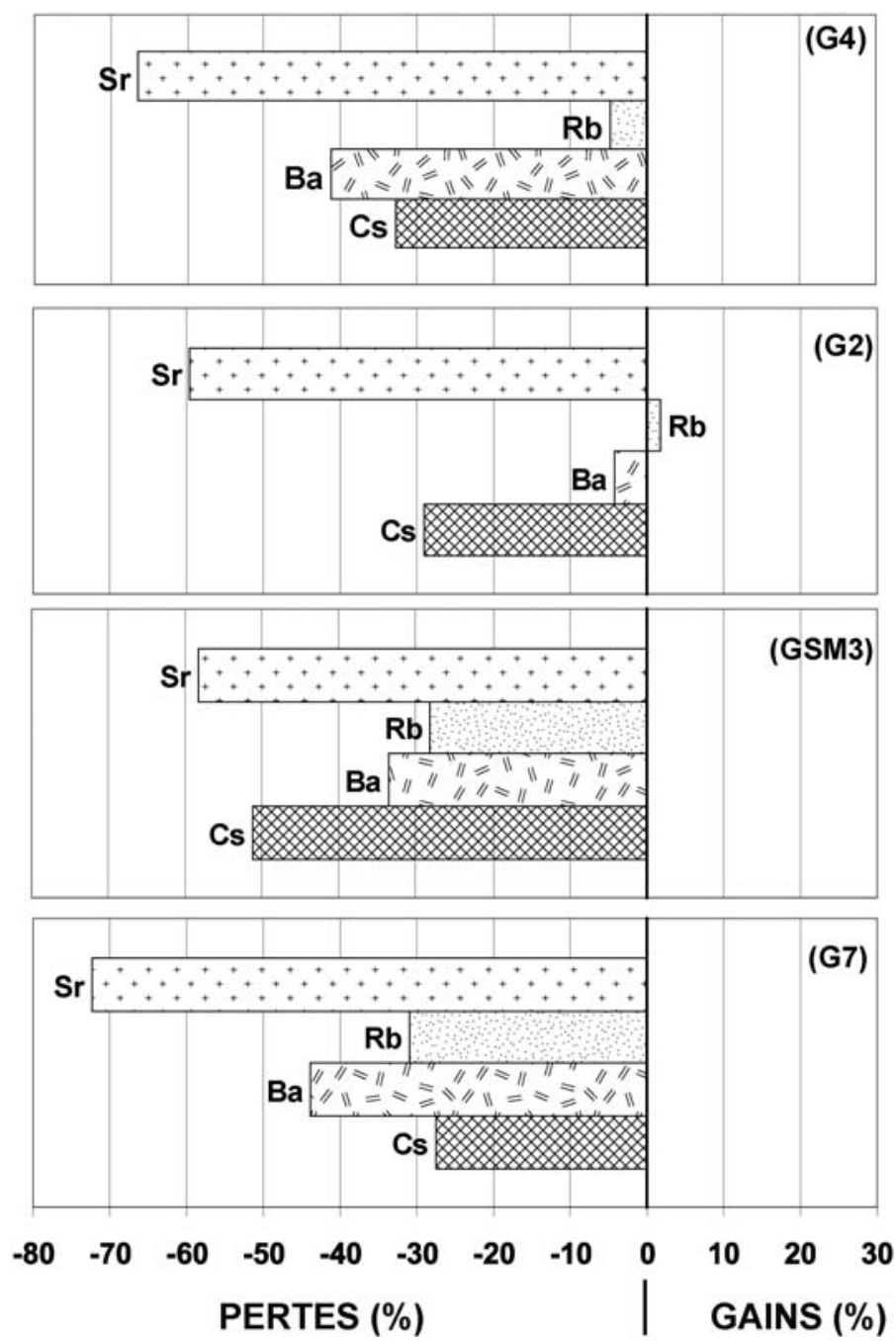

(b)

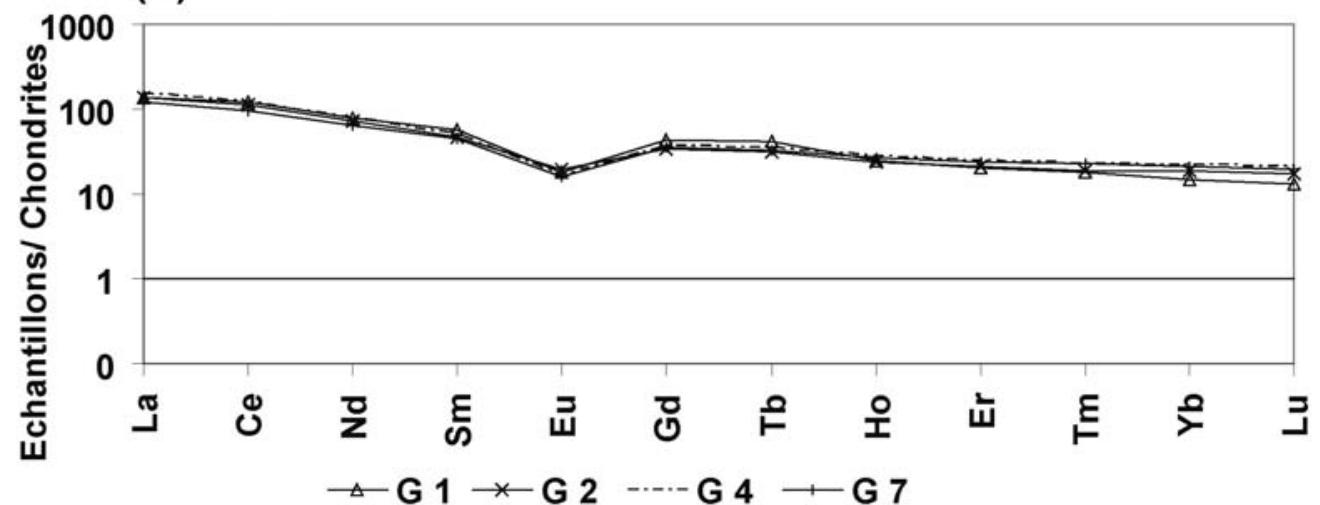

Fig. 7.-Distribution des éléments traces au cours de l'altération météorique des diatexites; (a) Diagrammes des gains et pertes en éléments traces, (b) Spectres des terres rares des diatexites du massif de l'Edough normalisées aux chondrites (Sun \& McDonough, 1989). La similitude dans l'allure des spectres reflète une source unique ainsi que des évolutions chimiques semblables pour tous les échantillons. 
concentrations habituellement rencontrées dans les roches de source acide (granitique) ou dans les roches riches en phases argileuses pouvant piéger ces éléments. Les spectres des terres rares (fig. 7b) normalisés aux chondrites (Sun \& McDonough 1989) présentent une allure en pente, avec un léger enrichissement en terres rares légères (LREE) par rapport aux terres rares lourdes (HREE). Eu/Sm varie de 0,11 à 0,15 . La similitude de l'allure des spectres reflète une source unique ainsi que des transformations épigénétiques semblables pour tout l'affleurement des diatexites.

\section{Conclusion}

Le profil chimique, les valeurs de CIA, CIW et IFRAIS et le taux de changement suggèrent que les diatexites ont subi une altération chimique modérée de type bisiallitisation. Le lessivage continu, sur un laps de temps long, a conduit à une évolution progressive dans la composition chimique des minéraux néoformés. La kaolinite s'est formée dans la partie sommitale de l'affleurement qui se caractérise par une forte fissuration ayant favorisé un bon drainage. Les smectites ne sont abondantes que dans la partie intermédiaire et basse des diatexites, qui est mal drainée. Les spectres des terres rares montrent un enrichissement en terres rares légères par rapport aux terres rares lourdes, ainsi qu'une similitude dans l'allure des patrons. Ces spectres traduisent une source unique quartzo-feldspathique ainsi que des transformations épigénétiques semblables. Ils confirment l'altération météorique des diatexites.

\section{REMERCIEMENTS}

L'auteur remercie le personnel des laboratoires du GFZ Potsdam, de l'Institut des Sciences de la Terre et de l'Environnement de l'Université de Potsdam (Allemagne) ainsi que ceux du laboratoire RX du CRD Boumerdes (Algérie). L'auteur remercie également les deux lecteurs pour leur contribution constructive.

\section{Références}

Ahmed Said, Y.; Leake, B.E. \& Rogers, G. (1993). The petrology, geochemistry and petrogenesis of the Edough igneous rocks, Annaba, NE Algeria. Journal of African Earth Sciences, 17(1): 111-123. doi:10.1016/0899-5362(93)90027-N
Baldeyrou, A.; Vidal O. \& Fritz B. (2003). Etude expérimentale des transformations de phases dans un gradient thermique:application au granite de Soultz-sousForêts, France. Comptes Rendus Géoscience, 335(4): 371-380. doi:10.1016/S1631-0713(03)00056-7

Gardner, L.R. (1980). Mobilization of Al and Ti during rock weathering isovolumetric geochemical evidence. Chemical Geology, 30: 151-165. doi:10.1016/00092541(80)90122-9

Gleizes, G.; Bouleton, J.; Bossière, G. \& Collomb, P. (1988). Données lithologiques et pétrostructurales nouvelles sur le Massif cristallophyllien de l'Edough (Est Algérien). Comptes Rendus de l'Académie des Sciences, Paris, series II, 306: 1001-1008.

Grabe-Schönberg, C.D. (1993). Simultaneous determination of thirty-seven trace elements in twenty eight international rock standards by ICP-MS. Geostandards Newsletter, 17(1): 81-98.

Grant, J.A. (1986). The isocon diagram - a simple solution of Gresens' equation for metasomatic alteration. Economic Geology. 81: 1976-1982. doi:10.2113/gsecongeo.81.8.1976

Gresens, R.L. (1967). Composition-volume relationships of metasomatism. Chemical Geology, 2: 47-65. doi:10.1016/0009-2541(67)90004-6

Hadj Zobir, S.; Laouar, R. \& Oberhänsli, R. (2007). Les métabasites de Sidi Mohamed (Edough, NE Algérien): Caractéristiques pétrographiques, minéralogiques et géochimiques. Bulletin Du Service Géologique National, 18(1): 25-41.

Hadj Zobir, S. (2008). Caractérisation du protolithe des diatexites du massif de l'Edough (Annaba, NE Algérien). Colloque International "Terre et Eau II", Annaba, Algérie.

Hammor, D. \& Lancelot, J. (1998). Métamorphisme miocène de granites panafricains dans le Massif de l'Edough (Nord-Est de l'Algérie). Comptes Rendus de l'Académie des Sciences, Paris, série II, 327: 391-396.

Harnois, L. (1988). The CIW index: a new chemical index of weathering. Sedimentary Geology, 55: 319322 .

Hilly, J. (1962). Etude géologique du Massif de l'Edough et du Cap de Fer (Est Constantinois). Publications du Service de la Carte Géologique de l'Algérie (nouvelle série) $19,408$.

McLennan, S.M.; Taylor, S.R. \& Kroner, A. (1983b). Geochemical evolution of Archean shales from South Africa 1. The Swaziland and Pongola Supergroups. Precambrian Research, 22: 93-124. doi:10.1016/03019268(83)90060-8

Middelburg, J.J.; Van Der Weijden, C.H. \& Woittiez, J.R.W. (1988). Chemical Processes affecting the mobility of major, minor and trace elements during weathering of granitic rocks. Chemical Geology, 68: 253-273. doi:10.1016/0009-2541(88)90025-3

Millot, G. (1964). Géologie des argiles. Masson et Cie, éd., Paris, 499 pp.

Nesbitt, H.W. (1979). Mobility and fractionation of rare earth elements during weathering of granodiorite, Nature, 279: 206-210. doi:10.1038/279206a0 
Nesbitt, H.W. \& Young, G.M. (1982). Early Proterozoic climate and plate motions inferred from major element chemistry of lutites. Nature, 299: 715-717. doi:10.1038/299715a0

Nesbitt, H.W. \& Young, G.M. (1984). Prediction of some weathering trends of plutonique and volcanic rocks based on thermodynamic and kinetic consideration. Geochimica et Cosmochimca Acta, 48: 15231534. doi:10.1016/0016-7037(84)90408-3

Oularbi, A. \& Zeghiche, A. (2009). Sensibilité à l'érosion du massif cristallophillien de l'Edough (Nord-Est Algérien). Synthèse, 20: 58-72.

Pédro, G. (1966). Essai sur la caractérisation géochimique des différents processus zonaux résultant de l'altération des roches superficielles (cycle aluminosilicique). Comptes Rendus de l'Académie des Sciences Paris, 262: 1828-1831.

Piché, M. (2000). Quantification de l'altération hydrothermale des roches du camp minier de Joutel à partir des analyses des éléments majeurs. Ministère des Ressources naturelles du Québec, MB-2000-06.

Piché, M. (2001). Atelier de géochimie - Une introduction à l'utilisation des minéraux normatifs pour quantifier l'altération hydrothermale associée aux gisements de SMV et aurifères. Association des Prospecteurs du Québec, 27e Congrès annuel, 11-13 septembre 2001, Val-d'Or, Québec. Cours intensif, 30 pp.

Plessen, H.G. (1997). Analytik und Geochemie der Platingruppenelemente in magmatischen Gesteinen. Scientific Technical Report STR97/11, Geoforschungszentrum, Potsdam, $135 \mathrm{pp}$.
Potdevin, J.L. \& Caron, J.M. (1986). Transferts de matière et déformation synmétamorphique pli. I:Structures et bilans de matière. Bulletin de Mineralogie. 109(4): 395-410.

Potdevin, J.L. \& Marquer, D. (1987). Méthodes de quantification des transferts de matière fluides dans les roches métamorphiques déformées. Geodinamica Acta 1(3): 193-206.

Sun, S. \& McDonough, W.F. (1989). Chemical and isotopic systematics of oceanic basalts; implications for mantle composition and processes. In: Magmatism in the Ocean Basins. (Saunders, A.D. \& Norry, M.J., Eds.). Geological Society of London, London, United Kingdom, 313-345.

Tardy, Y. (1969). Géochimie des altérations. Étude des arènes et des eaux de quelques massifs cristallins d'Europe et d'Afrique. Thèse de Doctorat d'État, Université Strasbourg, Mémoires du Service de la Carte géologique. Alsace-Lorraine, n 31, 199 pp.

Wronkiewicz, D.J. \& Condie, K.C. (1987). Geochemistry of Archean shales from the Witwatersrand Supergroup, South Africa: source-area weathering and provenance. Geochimica et Cosmochimca Acta, 51: 2401-2416. doi:10.1016/0016-7037(87)90293-6

Zuleger, E. \& Erzinger, J. (1988). Determination of the REE and Y in silicate materials with ICP-AES. Analytical Chemistry, 332(2): 140-143. doi:10.1007/BF00470631

Recibido el 10 de marzo de 2011

Aceptado el 28 de febrero de 2012

Publicado online el 12 de julio de 2012 